\title{
La mondialisation dans les programmes de SES
}

\section{Alain Beitone}

\section{OpenEdition}

Journals

Édition électronique

URL : http://journals.openedition.org/trema/3089

DOI : 10.4000/trema.3089

ISSN : 2107-0997

\section{Éditeur}

Faculté d'Éducation de l'université de Montpellier

\section{Édition imprimée}

Date de publication : 1 décembre 2013

Pagination : 76 - 89

ISSN : 1167-315X

\section{Référence électronique}

Alain Beitone, «La mondialisation dans les programmes de SES », Tréma [En ligne], 40 | 2013, mis en ligne le 01 décembre 2015, consulté le 30 avril 2019. URL : http://journals.openedition.org/trema/3089 ; DOI : 10.4000/trema.3089

Ce document a été généré automatiquement le 30 avril 2019

Trema 


\title{
La mondialisation dans les programmes de SES
}

\author{
Alain Beitone
}

1 L'étude du contenu des programmes d'enseignement occupe une place centrale dans la réflexion sur l'éducation (sociologie des savoirs scolaires, histoire de l'éducation, didactiques disciplinaires, etc.). Nous étudierons ici le cas de la mondialisation dans les programmes de sciences économiques et sociales (SES) de l'enseignement secondaire français ${ }^{1}$. Il faut souligner que ce thème est par ailleurs présent dans les programmes d'histoire et de géographie et dans les programmes d'économie des filières technologiques tertiaires. Une étude comparée du traitement de la mondialisation dans ces divers programmes serait du plus haut intérêt. Mais nous adoptons ici un point de vue restreint à une seule discipline.

2 Les programmes scolaires et leur évolution peuvent être appréhendés à partir de trois grandes grilles de lecture

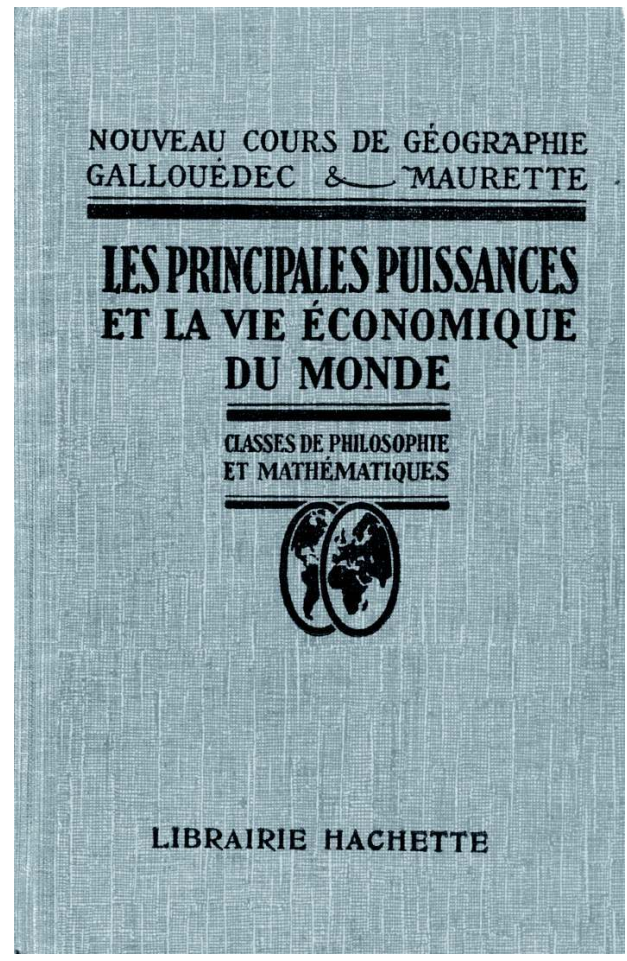
(interdépendantes). Premièrement, les programmes étant une transposition des savoirs savants, nous nous demanderons quels sont les liens entre l'évolution des savoirs de référence et l'évolution du traitement de la mondialisation dans les programmes. Deuxièmement, les programmes sont liés à la mobilisation de diverses catégories d'acteurs (la noosphère au sens d'Y. Chevallard). Au cœur des controverses se trouve la référence à la notion de «question vive » et la question de la mobilisation de diverses 
disciplines des sciences sociales à propos de la mondialisation. Enfin, la question de l'enseignabilité des savoirs, joue aussi un rôle important.

\section{La mondialisation : des savoirs savants aux savoirs à enseigner}

\section{I.1. Les premiers programmes de SES : la mondialisation absente}

3 La discipline scolaire « Sciences économiques et sociales » a pris naissance en 1966-1967 avec la «Réforme Fouchet» (Chatel et alii 1990, Beitone et alii 2013). Les premiers programmes sont rédigés par un géographe et un historien (Marcel Roncayolo et Guy Palmade). La notion de mondialisation n'y figure pas. Les questions internationales sont présentées ainsi dans le programme initial de la discipline :

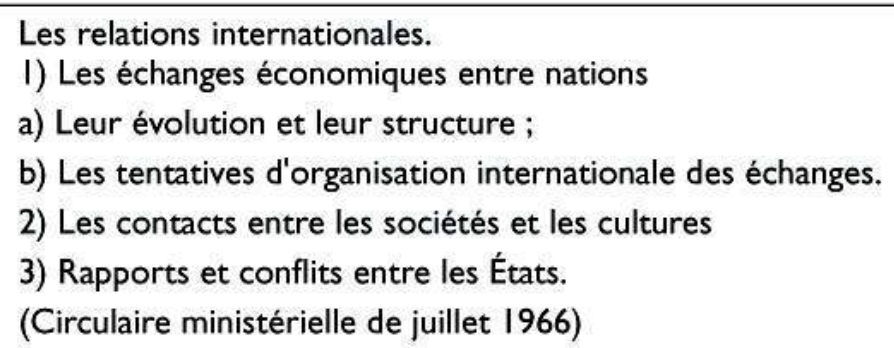

On notera (mais nous y reviendrons) que les trois dimensions économique, sociologique et science politique (relations internationales) sont présentes.

Sur le plan économique c'est la dimension internationale qui est mise en avant. À cette époque d'ailleurs le programme de première est centré sur l'économie nationale et celui de terminale sur la croissance, le changement social et les relations internationales. L'articulation du national et de l'international est donc centrale et la mondialisation est absente.

6 Les relations économiques sont donc d'abord conçues comme nationales et, dans un second temps on envisage l'impact des échanges internationaux. Cela est conforme à la façon dont le commerce international est étudié en science économique depuis Adam Smith et David Ricardo. C'est aussi conforme à la façon dont la régulation économique nationale et internationale est pensée après la Seconde guerre mondiale. Qu'il s'agisse de commerce (GATT), de monnaie et de finance (FMI), de culture (UNESCO), de politique (ONU), ce sont les relations inter étatiques qui sont mises au premier plan.

7 On peut noter cependant que certains éléments du savoir savant ne sont pas pris en compte dans ces programmes initiaux. En particulier les approches développées dès les années 1950 par Maurice Byé ${ }^{2}$ en termes de "grande unité interterritoriale » ou les approches de François Perroux ne sont pas évoquées dans le programme. En 1960, Stephen Hymer (qui sera considéré par la suite comme un précurseur de l'étude de la multinationalisation des firmes) soutient sa thèse au MIT (voir aussi Hymer, 1968). Ce type d'approche n'est pas non plus présent dans les programmes. Pas plus que ne seront prises en compte ultérieurement les approches hétérodoxes de Christian Palloix qui raisonne en termes d'économie mondiale dès les années 1970 (Palloix, 1971). On trouve ici la confirmation du fait que le processus de transposition didactique repose, entre autres, 
sur un processus de sélection des savoirs de référence. Processus qui s'étend généralement sur une longue période.

8 La deuxième grande génération de programme, celle de 1982, ne comporte pas non plus de référence au concept de mondialisation.

- L'internationalisation de la production et des échanges ;

- La division internationale du travail ;

- Le système monétaire international.

(Arrêté du 9 mars 1982)

9 C'est d'autant plus étonnant que, selon une étude du FMI, le terme « mondialisation » est couramment utilisé depuis les années 1980 (FMI, 2000).

\section{2. Emergence du concept dans les savoirs savants et absence dans les programmes}

10 On considère généralement que c'est avec Marshall Mc Luhan (1968) et le thème du « village global » que la référence à la mondialisation (« globalisation » est un anglicisme) commence à se développer. Dans le champ de l'analyse économique, l'article de Theodor Levitt (1983) est considéré comme précurseur. L'ouvrage de Kenichi Ohmae (1985) va aussi avoir un retentissement important. En France le Groupement d'intérêt scientifique pour l'étude de la mondialisation et du développement (GEMDEV) est créé en 1983.

11 Mais c'est surtout dans les années 1990 que les publications et les évènements se multiplient.

12 En 1990 parait le livre de Michel Aglietta, Anton Brender et Virginie Coudert sur la globalisation financière. Ce livre reste une référence incontournable sur le sujet. En 1991 paraît aux États-Unis le livre de Robert Reich, The Work of Nations, traduit en français en 1993 sous le titre L'économie mondialisée. Le livre a un retentissement considérable (il est traduit dans plus de 20 langues). En France Jacques Adda publie en 1996 l'ouvrage de synthèse sur La mondialisation de l'économie (livre constamment réédité depuis). La même année, François Chesnais dirige chez Syros un ouvrage collectif sur la globalisation financière. Autre ouvrage collectif qui fera date, celui que dirige Serge Cordellier à la Découverte : La mondialisation au-delà des mythes, avec des contributions de Dominique Plihon et Robert Boyer notamment. Le mouvement Attac est créé en 1998. Le Groupe d'études et de recherches sur les mondialisations (GERM, association loi de 1901) est constitué en 1999.

L'accroissement spectaculaire du recours au terme "mondialisation » est bien souligné par René Dagorn (1999, p. 197) : «En 1991 puis en 1995, la Documentation française consacre deux numéros des Cahiers français à l'étude du commerce international. En 1991, l'éditorial ne comporte aucune utilisation du mot «mondialisation» et dix fois le mot « international». Cinq ans plus tard, le second éditorial comprend dix-sept fois le mot « mondialisation » et seulement trois fois le mot international».

Pourtant, le programme de SES de 1994 ne fait toujours pas référence à la mondialisation 
L'ouverture internationale

- Libération des échanges et développement ;

- Protectionnisme et développement;

- Le règlement des échanges et l'endettement.

(Arrêté du 2 septembre 1994) est structuré par le débat libre-échange/protectionnisme (donc les rapports entre nations). La question de l'internationalisation des systèmes productifs (vocabulaire plutôt marxiste) ou de l'émergence de firmes globales (vocabulaire plutôt orthodoxe) n'est pas prise en compte dans le programme. Alors qu'il est question d'une crise de la dette (des États) depuis le début des années 1980 (crise de la dette mexicaine de 1982), le thème est introduit dans le programme, mais pas le phénomène de la mondialisation dans son ensemble (et notamment pas la globalisation financière). La perspective tiers-mondiste est toujours présente. Dans la troisième colonne du programme («indications complémentaires»), les concepteurs du programme précisent à propos du thème «Libération des échanges et développement : « On examinera les problèmes de l'insertion des pays en développement dans les échanges internationaux ». C'est une référence à des débats anciens sur le développement autocentré ou sur la détérioration séculaire des termes de l'échange.

\section{I.3 L'entrée de la mondialisation dans les programmes de SES}

C'est avec le programme de 1998 que la mondialisation fait son entrée dans les programmes de SES.

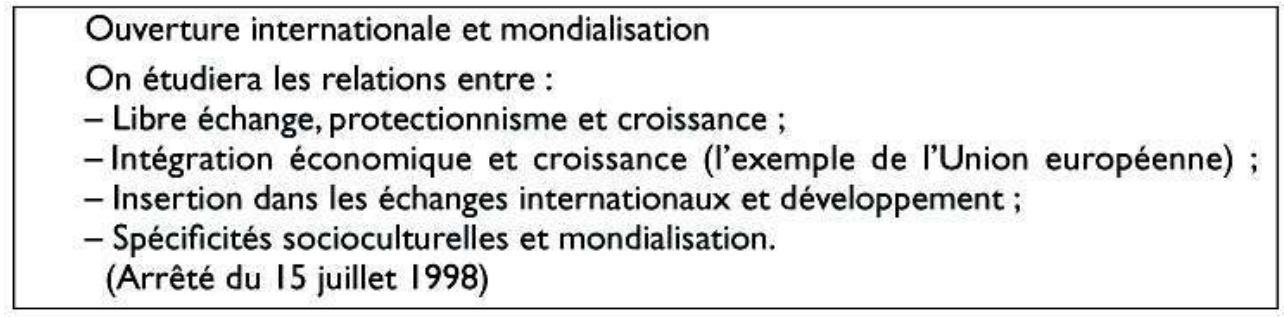

Plusieurs remarques s'imposent à propos de ce nouveau programme. Si le mot mondialisation figure bien dans le titre de la sous partie du programme, ce concept n'est pas vraiment pris en compte. En effet, les trois premiers points de cette sous-partie renvoie (comme précédemment) aux questions de libre échange et de protectionnisme. La dimension tiers-mondiste se traduit par la présence (dans les notions essentielles et les notions complémentaires) des termes suivants : développement extraverti, dépendance. De même, le troisième point de cette sous-partie «insertion dans les échanges internationaux et développement » renvoie aux débats des années 1960 et au choix entre développement autonome et développement extraverti. La véritable nouveauté réside ici dans l'introduction de l'intégration européenne : il est vrai que nous sommes à la veille du passage à l'euro.

Le terme mondialisation, quant à lui, n'apparait qu'à propos des spécificités culturelles. Il fait référence au débat relatif à l'éventuelle remise en cause des spécificités culturelles nationales par la mondialisation (d'où le terme d'«acculturation" dans la liste des notions que les élèves doivent acquérir). Si ce débat est important ${ }^{3}$ il ne constitue qu'un 
aspect des débats sur la mondialisation. Par exemple, la question des délocalisations, celle de la montée des pays émergents ou encore celle de l'impact de la mondialisation sur l'emploi ne sont pas évoqués. De même, si les « notions complémentaires » (3e colonne du programme) comportent les termes «taux de change, régime de change et marchés financiers », la globalisation financière n'est toujours pas citée.

Le programme de 2002, même s'il apparait, en première approche, dans la continuité du précédent, marque cependant des inflexions importantes.

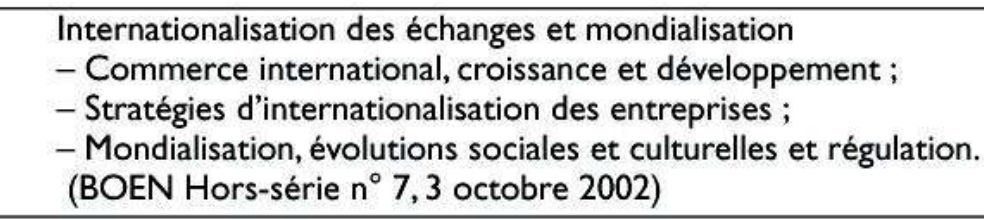

Tout d'abord, dans l'intitulé de la sous-partie, on passe de l'idée d'ouverture internationale (qui suppose une situation préalable d'économie fermée) à l'étude du processus d'internationalisation. Le point 2 de cette sous partie est consacré aux "stratégies d'internationalisation des entreprises" et on trouve dans la colonne 1 (notions essentielles que les élèves doivent acquérir) le terme "firmes transnationales". Le débat sur la place respective des firmes et des Etats-nations est donc posé. Dans les notions complémentaires on trouve les termes «commerce intra firme» et « délocalisation ». Les indications complémentaires invitent les professeurs à étudier « les conditions dans lesquelles les entreprises transnationales mettent en concurrence territoires et États ». La troisième partie ne se limite plus à la question culturelle mais introduit la notion de régulation. Si le terme " gouvernance mondiale » n'est pas présent, les indications complémentaires précisent : «On mettra en évidence les effets induits par la mondialisation dans les modes de régulation de l'économie et de la société ainsi que dans la distribution des rôles entre États et marchés. On montrera que de nouvelles formes de coordination et d'institutionnalisation émergent au niveau international (action internationale des syndicats, des autres ONG, des institutions internationales). On discutera des questions suscitées par des propositions d'édicter des normes sociales et environnementales applicables à l'échelle mondiale ». C'est donc la naissance d'un espace public mondial qui est prise en compte, de même que la nécessité de mettre en place des institutions et des procédures de régulation qui dépassent le cadre national.

Ces inflexions, qui conduisent à introduire véritablement la question de la mondialisation dans les programmes (quoi que de façon assez prudente) doivent dans une large mesure être mises en relation avec la présence dans le groupe d'experts de deux économistes très impliqués dans ce champ de recherche (et tous deux économistes à l'OFCE). Jean-Luc Gaffard, le président du groupe, travaille sur la question de l'innovation, des marchés, des stratégies d'entreprises, du système productif. Jacques Le Cacheux publie en mars 2002, un article important sur la mondialisation (rédigé par conséquent alors même qu'il participait aux travaux du groupe d'experts qui a rédigé le programme).

La dernière génération de programmes (entrée en vigueur en septembre 2012 en classe de terminale) a suscité de nombreux débats que nous ne traiterons pas ici. Pour nous en tenir à notre objet d'étude, on constate que le groupe d'experts (présidé par Jacques Le Cacheux) a prolongé les évolutions antérieures : 
Mondialisation, finance internationale et intégration européenne

- Quels sont les fondements du commerce international et de l'internationalisation de la production ?

- Comment s'opère le financement de l'économie mondiale?

- Quelle est la place de l'Union européenne dans l'économie globale ? (BO spécial $n^{\circ} 8,13$ octobre 2011)

On notera tout d'abord que, pour la première fois, le terme « mondialisation » apparaît en tête de l'intitulé de cette partie du programme. Assez logiquement, le commerce international est rapproché de l'internationalisation de la production. Les indications complémentaires parlent à ce propos de la «mondialisation de la production ». Le second point de cette partie du programme porte sur le financement de l'économie mondiale. Celle-ci est donc considérée comme une réalité qu'il convient d'étudier en tant que telle. Le troisième point invite les professeurs à étudier l'articulation entre la construction européenne et «l'économie globale ».

Une des innovations de ce nouveau programme est la création d'un enseignement d'économie approfondie. L'un des trois thèmes de cet enseignement porte sur la globalisation financière: c'est la première fois que ce terme figure dans les programmes de SES. Les deux autres thèmes prennent aussi en compte la mondialisation. L'un porte sur les rapports entre démographie et économie et analyse la mondialisation des flux d'épargne liée à la différence de la situation des divers pays en ce qui concerne le cycle de vie. L'autre porte sur les stratégies d'entreprise et la concurrence dans une " économie globalisée ». Le programme de 2011 considère donc la mondialisation comme un fait et les divers points étudiés le sont dans le cadre d'une économie mondialisée.

Il s'est donc écoulé entre dix et vingt ans entre le moment où le thème de la mondialisation acquiert une place importante dans les savoirs savants et le moment où ce concept trouve véritablement sa place dans les programmes de SES. Ce décalage temporel n'a rien de surprenant au regard de la théorie de la transposition didactique. Rappelons que les fameuses "mathématiques modernes" dont l'introduction dans les programmes de l'enseignement secondaire dans les années 1960 déclencha de très vifs débats dataient en réalité des travaux d'Evariste Gallois au XIXe siècle. Pour qu'un savoir savant soit transposé en savoir à enseigner, il est nécessaire qu'il soit suffisamment robuste sur le plan scientifique, qu'il soit suffisamment diffusé au-delà des seuls milieux académiques (revues de vulgarisation, enseignement supérieur, etc.), qu'il ait fait l'objet d'une restructuration permettant son appropriation par les élèves et son intégration dans l'ensemble des programmes scolaires. On sait aussi que l'intégration d'un savoir nouveau suscite des réticences (voire une hostilité) de la part des enseignants, les responsables de l'institution éducative sont, de ce fait, manifestement soucieux d'une intégration progressive : chaque nouveau programme doit être suffisamment proche du programme précédent pour continuer à être familier aux enseignants ${ }^{4}$. L'introduction du concept de mondialisation dans les programmes de SES relève manifestement de cette logique de glissement progressif qui conduit à occulter en partie les enjeux du concept au sein des savoirs savants (et donc les débats parfois vifs qu'il suscite). Signalons enfin le rôle décisif que jouent dans certains cas les universitaires qui sont associés à la rédaction des programmes. Du fait de leur intégration dans le champ de la recherche, ils ont conscience de l'évolution du savoir savant et souhaitent son introduction dans les programmes scolaires. Par exemple, lors du séminaire national sur les programmes scolaires organisé par le DESCO en mars 2004 à Lyon, J. Le Cacheux insistait sur les nombreuses et 
importantes avancées de la théorie économique et il jugeait nécessaire de combiner l'enseignement des savoirs les plus anciennement constitués au sein de la discipline en même temps que le renouvellement très important du savoir économique (il n'hésite pas à parler de « révolutions $»^{5}$ ).

L'exemple de la mondialisation permet donc de mettre en évidence une tension qui caractérise l'ensemble des programmes scolaires : il existe d'une part une certaine inertie liée à la nécessité de ne pas introduire des changements trop importants par rapport à l'existant, d'autre part une évolution des savoirs savants qui appellent à des remises en causes parfois radicales.

\section{La mondialisation : question vive et savoir enseignable}

27 Les savoirs scolaires sont confrontés à une autre exigence que celle de leurs rapports avec les savoirs savants. Ils doivent s'articuler (d'une façon qu'il convient de préciser) avec les débats qui traversent la société. Les historiens sont confrontés aux diverses mémoires parfois en conflit, les professeurs de SVT aux débats sur le genre, sur la sexualité, les professeurs de physique aux débats sur le nucléaire et les nanoparticules, etc.

\section{1. La mondialisation : entre scientisme et relativisme}

Les SES n'échappent pas à cette exigence et à ces problèmes ${ }^{6}$. Certains milieux patronaux ont reproché aux professeurs de SES d'adhérer aux thèses altermondialistes. Et on a pu à l'inverse reprocher à l'institut de l'entreprise d'adopter, lors des premiers «Entretiens Louis-le-Grand » (octobre 2003) une vision apologétique des rapports entre entreprises et mondialisation ${ }^{7}$. À cette occasion, certains enseignants avaient demandé que, concurremment à ce stage organisé avec l'Institut de l'entreprise, le ministère en organise un autre en liaison avec l'association ATTAC. Cela revient à considérer l'enseignement de telles questions vives comme la confrontation d'opinions. Le débat d'opinion deviendrait presque une fin en soi. Du coup, la question des savoirs est évacuée ou marginalisée.

Entre un scientisme selon lequel il n'existe qu'un discours légitime et un relativisme qui considère qu'il n'existe pas de savoir établi du tout mais seulement des points de vue inconciliables entre eux, les deux derniers groupes d'experts ont tracé une voie médiane, sur la mondialisation, comme sur les autres points du programme.

S'agissant de la mondialisation, J. Le Cacheux posait le problème d'emblée dans son article de mars 2002: «Bénéfique pour certains, maléfique pour d'autres, la mondialisation est tantôt parée de toutes les vertus, tantôt affublée de tous les vices ». Établissant le lien avec les débats politiques et les conflits sociaux, J. Le Cacheux précisait: "À Gênes, à l'occasion du sommet du G8 à l'automne 2000, comme à Barcelone en mars 2002 lors du récent sommet européen, les responsables politiques vantaient, dans des enceintes luxueuses protégées comme des forteresses assiégées, les bienfaits d'une ouverture et d'une libéralisation qu'ils proposaient d'accentuer, tandis que les opposants manifestaient dans les rues alentour. Partisans et détracteurs de la mondialisation adoptent volontiers, dans les débats, des positions extrêmes et caricaturales » (Le Cacheux, 2002, p. 21). Refusant cette alternative, il précisait d'abord quels sont les 
principaux moteurs de la mondialisation: "avantages comparatifs et spécialisation, économies d'échelle et de gamme, goût des consommateurs riches pour la variété, abaissement des coûts de transport et de communication, et libéralisation et ouverture des marchés ». On peut considérer, a minima, que les élèves doivent connaître ces facteurs explicatifs et l'interdépendance entre eux. J. Le Cacheux ajoutait: «La mondialisation qui résulte de la combinaison de ces facteurs procure des gains économiques indéniables, gains mutuels de l'échange, gains de la spécialisation et diffusion internationale des technologies, qui sont susceptibles d'engendrer, à très long terme, une tendance au rattrapage des pays les plus riches par les plus pauvres, donc une certaine convergence économique mondiale. Toutefois, la mondialisation économique et financière comporte aussi des coûts et des inconvénients, notamment en raison des restructurations qu'elle impose, de l'instabilité macroéconomique et financière qu'elle favorise en l'absence de régulations adéquates, et des inégalités qu'elle creuse entre gagnants et perdants, au sein des économies nationales et, dans certaines conditions, entre économies nationales ". (Le Cacheux, 2002, p. 19). Il existe en effet un certain nombre de travaux robustes (ceux de Stiglitz, Krugman, Sachs, Rodrik, Bourguignon, Cohen, Michalet, etc.) qui peuvent servir utilement de base à un enseignement rigoureux et nuancé à propos de la mondialisation. Il ne s'agit en aucun cas d'esquiver les débats, mais de s'en tenir aux débats scientifiques et aux règles qui les régissent (seule doit prévaloir la force du meilleur argument écrivait P. Bourdieu). L'institution scolaire n'est pas le café du commerce. Elle est le lieu où les élèves doivent pouvoir s'approprier des savoirs qui disposent d'une légitimité savante. Le professeur n'est pas dans la classe pour favoriser la confrontation des opinions, il est le garant de la validité épistémologique des savoirs à l'œuvre au sein de l'école.

31 De façon plus générale, J.L. Gaffard définissait, lors d'une intervention aux Journées de l'économie de Lyon, une posture à propos de la science économique qui pourrait utilement servir de référence pour l'enseignement des SES (et de la mondialisation en particulier). Le premier point rejette le relativisme (la science économique n'est pas un ensemble d'opinions), le second point rejette le scientisme: il n'existe pas de discours économique qui pourrait s'imposer ou se substituer aux indispensables choix politiques. Le troisième point indique la fonction du discours économique : éclairer les choix par des savoirs obéissant aux règles de la production scientifique. De même que l'expert doit fournir aux décideurs politiques des connaissances susceptibles d'éclairer leur choix, le professeur de SES doit fournir aux élèves les armes intellectuelles de choix citoyens éclairés. Mais l'espace de la classe n'est pas le lieu du débat sur les choix citoyens, il est le lieu de l'appropriation des savoirs qui permettent d'agir en citoyen.

La science économique selon J.L. Gaffard

«- L'économie n'est pas une collection d'opinions toutes équivalentes et exprimant des préférences idéologiques.

- Pour autant l'analyse économique n'a pas pour enjeu d'édicter des règles définies par les savants et applicables par les décideurs.

- Elle doit éclairer des arbitrages forcément politiques entre des objectifs contradictoires ou conciliant des déséquilibres ».

J.L. Gaffard, « Enseignement et recherche en économie : le défi ». Intervention lors des journées de l'économie, Lyon 20, 21, 22 novembre 2008.

Ce double refus du scientisme et du relativisme se retrouve dans le préambule du programme du cycle terminal actuellement en vigueur: «L'accent mis sur l'ambition 
scientifique des sciences sociales ne doit pas conduire cependant à un point de vue "scientiste ». L'activité scientifique ne consiste pas à construire un ensemble de dogmes ou de vérités définitives, mais à formuler des problèmes susceptibles d'être résolus par le recours au raisonnement théorique et à l'investigation empirique. Pour autant, la posture scientifique conduit à refuser le relativisme : tous les discours ne se valent pas et rien ne serait pire que de donner l'impression aux élèves que «les sciences sociales ne sont qu'une collection d'opinions contradictoires sur le monde, qui, au final, se valent toutes et donc ne permettent de fonder aucun savoir solide » (rapport de la commission présidée par Roger Guesnerie, professeur au Collège de France) » (BO spécial n 9 du 30 septembre 2010).

\section{2. La mondialisation : un fait social total ?}

33 Comme nous l'avons souligné plus haut, les premiers programmes de SES (ceux de 1966) comportaient une dimension " économique ", mais aussi une dimension "sociologique " et une dimension relevant de la science politique. Mais, dès 1974, à l'occasion des allègements de programme liés à la mise en place des «10\%" par le ministre de l'éducation Joseph Fontanet, la dimension sociologique et la dimension science politique disparaissent. Depuis cette époque, les points du programme qui concernent les relations économiques internationales puis la mondialisation sont abordées presque exclusivement sous l'angle de la science économique (commerce international, protectionnisme, libre échange, etc.). Certes un problème "social» est présent («les spécificités socioculturelles » dans le programme de 1998, «Évolutions sociales et culturelles» dans le programme 2002), mais l'approche proprement sociologique de la mondialisation est très largement absente de même que l'approche anthropologique et l'approche relevant de la science politique.

Ce point est important à souligner car une certaine doxa des $\mathrm{SES}^{8}$ affirme que depuis toujours, en SES, on refuse de pratiquer la spécialisation disciplinaire et on croise systématiquement les regards disciplinaires à partir d'une approche fondée sur les objets. Or l'étude des questions internationales et de la mondialisation montre qu'il n'en est rien. Depuis l'origine, c'est l'approche économique qui est clairement dominante (voire exclusive) dans les programmes, alors que dans le même temps, sur d'autres thèmes (la famille, la socialisation, les classes sociales, le pouvoir politique, etc.), ce sont clairement les approches qui relèvent de la sociologie et de la science politique qui ont été dominantes9. La spécialisation disciplinaire, plus explicite dans les programmes les plus récents, n'est pas une innovation radicale : elle se situe dans la continuité de pratiques anciennes au niveau des programmes comme des manuels. Comment expliquer cette spécialisation alors que certains professeurs influents, relayés par une association et plusieurs syndicats, se disent très attachés à la dimension «interdisciplinaire »? Une première explication réside dans le processus de la transposition didactique. Les analyses sociologiques de la mondialisation (Appadurai, 1990; Barber, 1996; Bauman, 1998, Martin, 2003 ; Beck, 2003 ; Bayard, 2004 ; Wagner, 2007 ; Abélès, 2008 ; Sassen, 2009) sont beaucoup plus récente ${ }^{10}$ Le premier livre de synthèse à vocation pédagogique est paru en 2013 (Lecler, 2013). Conformément à ce que nous écrivions plus haut, il s'écoule toujours du temps entre le développement du savoir savant et sa transposition didactique.

Mais la raison essentielle n'est sans doute pas là. De plus en plus nettement au cours du temps, et de façon explicite dans la dernière génération de programmes, l'objectif des SES 
est d'initier les élèves aux acquis de trois disciplines des sciences sociales (science économique, sociologie et science politique) qui ne sont pas enseignées dans d'autres disciplines scolaires. Le point de départ de la conception des programmes est donc la spécificité des regards disciplinaires (et non les objets d'études qui par définition peuvent être étudiés $d u$ point de vue de très nombreuses disciplines ${ }^{11}$ ). S'agissant de la mondialisation, les concepteurs des programmes ont clairement opté pour la lecture de la science économique.

La mondialisation est donc un objet d'étude à l'occasion duquel on fait travailler les élèves sur des raisonnements (le gain à l'échange, la spécialisation, la concurrence, les règles qui encadrent les échanges marchands, etc.) qui sont spécifiques aux économistes. Sur d'autres points du programme, c'est la grille de lecture sociologique qui est mobilisée (par exemple sur la structure sociale, sur les pratiques culturelles, etc.).

Sur la mondialisation, comme sur de nombreux autres thèmes, la spécialisation disciplinaire est la règle, et pas seulement dans les derniers programmes. Ceux-ci ont spécifié que sur certains points particuliers, il fallait former les élèves au croisement des regards disciplinaires. Ce qui suppose que les élèves disposent d'une connaissance suffisante des regards disciplinaires. En général ce sont ces « regards croisés» que les professeurs ont trouvé les plus difficiles à traiter dans les nouveaux programmes. Indice sans doute que, dans la pratique des classes, la spécialisation disciplinaire est depuis longtemps la règle.

\section{3. Mondialisation et enseignabilité : l'exemple de la finance}

La question de l'enseignabilité des savoirs est évidemment capitale. Inutile de prévoir dans les programmes d'enseignements l'étude de questions qui seraient totalement hors de portée des élèves. On entend traditionnellement à propos des programmes deux types de discours. D'une part, certains dénoncent leur abstraction, leur encyclopédisme, leur caractère trop conceptuel, leur ambition déraisonnable. D'autre part, on demande aux disciplines scolaires (et particulièrement aux SES) de permettre la compréhension du monde, de former le citoyen, de rendre accessibles les grands débats relatifs aux questions économiques et sociales. Ces deux discours, bien que souvent tenus par les mêmes acteurs des débats sur les programmes, sont pourtant contradictoires. Le monde est en effet extraordinairement complexe. Pour le comprendre dans toute sa complexité il faut une haute maîtrise de savoirs théoriques et conceptuels. La mondialisation, et plus particulièrement la globalisation financière, sont un bon exemple de cette difficulté.

Un premier exemple peut être donné par le concept de "spread» (ou écart de taux d'intérêt). Ce concept avait été introduit par le groupe d'experts dans la première version du projet de programme de la classe de première dans la partie consacrée aux questions de monnaie et de crédit. Cela a provoqué, à l'occasion de la consultation sur le projet de programme, une levée de boucliers de certains enseignants, de l'association professionnelle (Association des Professeurs de Sciences Economiques et Sociales, APSES) et de certains syndicats. Le concept a donc été retiré du programme. Ce concept était nouveau, certes. Mais était-il complexe? C'est une simple soustraction si l'Allemagne emprunte à $2 \%$ d'intérêt sur 10 ans quand la Grèce emprunte à $20 \%$ le « spread » est de 18 points de pourcentage. Le calcul $(20-2=18)$ doit-il vraiment être considéré comme hors de portée d'un élève de première de l'enseignement général ? Ce concept est-il de nature à améliorer la compréhension du monde des élèves ? À l'évidence oui. De très nombreux 
quotidiens ont publié le graphique fameux montrant la convergence des taux d'intérêt obtenu après le passage à l'euro, puis le creusement spectaculaire de ces écarts de taux avec la crise des dettes souveraines en Europe. F. Fillon, alors premier ministre, a déclaré que le premier indicateur dont il prenait connaissance chaque matin était le "spread» entre la France et l'Allemagne. Ce creusement du différentiel de taux permet de faire le lien avec l'idée de « risque de crédit » qui figure aussi au programme. Les élèves sont-ils vraiment incapables de comprendre qu'un prêteur demande un taux d'intérêt plus élevé à un agent qui présente selon lui un risque de crédit plus important ? À partir de là, l'envol des taux d'intérêt sur les pays du sud de la zone euro est un symptôme de l'insuffisante solidarité au sein de la zone. On constate d'ailleurs que le spread se réduit chaque fois que les autorités européennes prennent des mesures suffisamment vigoureuses pour venir en aide aux pays qui font l'objet d'attaques spéculatives. Les auteurs qui ont consacré des ouvrages à la crise de la dette dans la zone Euro ont tous mis l'accent sur cet écart de taux (Artus et Gravet, 2012,; Aglietta, 2012 ; Pisani-Ferry, 2011). On voit par conséquent que cette suppression du concept d'écart de taux d'intérêt prive les élèves d'un outil précieux de compréhension du monde et ampute la "formation du citoyen " pourtant chère à de nombreux professeurs de SES. Au nom de la simplicité, on a donc réduit la compréhension du monde.

Le même problème s'est posé, de façon encore plus spectaculaire, en classe terminale. Suite aux revendications de l'association professionnelle, un allègement des programmes a été mis en place avec la participation active de responsables de cette association. Cette dernière met fortement l'accent sur la « formation du citoyen » et sur « l'esprit critique ». Or, sur la globalisation financière, l'allègement du programme conduit à supprimer $d u$ programme de tronc commun les concepts de "devises", "marché des changes " et "spéculation ». Dans le programme de l'enseignement de spécialité, on a supprimé des indications complémentaires les références au "contrôle de la finance dérégulée », aux "paradis fiscaux", aux "fonds spéculatifs». Ainsi, alors que nous traversons la plus grave crise financière depuis 1929, alors que les questions relatives au marché des changes (cours de l'euro, du dollar, du yuan, ...) font la une de l'actualité, alors que la question de la régulation financière est à l'ordre du jour des $G 20$ et suscite la mobilisation de la société civile mondiale, on supprime ces concepts du programme! La contradiction est frappante avec la volonté de formation du citoyen. Mais surtout cette démarche repose sur l'idée selon laquelle pour rendre l'accès au savoir plus égalitaire, il faudrait en rabattre sur le caractère conceptuel, théorique, ambitieux des programmes. Pourtant de nombreux travaux (ceux de J.P. Terrail notamment) soulignent qu'au contraire, il y a là une source de creusement des inégalités. Certains élèves pourront se voir expliquer ailleurs (dans leur famille ou dans les cours de certaines classes dans certains lycées) ce que sont la spéculation et le marché des changes et pourquoi de très nombreux économistes insistent sur la nécessité d'un contrôle accru de la finance dérégulée. Et d'autres seront privés de ces informations.

41 Certes il n'est pas possible de tout enseigner. Des choix sont incontournables. Mais on pourrait espérer deux choses :

- $\circ$ que les choix relatifs aux contenus des programmes reposent sur une évaluation sérieuse de l'enseignabilité des savoirs (et non sur des impressions de certains professeurs ou groupes de pression);

- que l'on s'efforce de mettre en cohérence l'ambition de compréhension du monde et les choix relatifs aux savoirs enseignés ${ }^{12}$. 


\section{BIBLIOGRAPHIE}

Abélès (Marc), Anthropologie de la globalisation, Payot, 2008.

Adda (Jacques), La mondialisation de l'économie, Paris, La Découverte, Coll. Repères, 1996.

Aglietta (Michel), Brender (Anton), Coudert (Virginie), Globalisation financière : l'aventure obligée, Paris, Economica, 1990.

Aglietta (Michel), Zone euro. Éclatement ou fédération, Paris, Michalon, 2012.

Appadurai (Arjun), Modernity at Large. Cultural Dimension of Globalization, University of Minnesota Press, 1990 (traduction française : Après le colonialisme. Les conséquences culturelles de la mondialisation, Paris, Payot, 2005.

Artus (Patrick) et Gravet (Isabelle), La crise de l'euro. Comprendre les causes, en sortir par de nouvelles institutions, Paris, Armand Colin, 2012.

Barber (Benjamin), Djihad versus Mcworld. Mondialisation et intégrisme contre la démocratie, Bruxelles, Desclée de Brouwer, 1996

Bauman (Zygmunt), Globalization. The Human Consequences, New York, Columbia University Press, 1998.

Bayart (Jean-François), Le gouvernement du monde. Une critique politique de la globalisation, Paris, Fayard, 2004.

Beck (Ulrich), Pouvoir et contre-pouvoir à l'ère de la mondialisation, Paris, Flammarion, 2003

Beitone (Alain) et alii, Les sciences économiques et sociales. Enseignement et apprentissages, Bruxelles, De Boeck, 2013.

Chatel (Elisabeth) et alii, Enseigner les sciences économiques et sociales. Le projet et son histoire, INRP, 1990. Disponible en ligne http://ses.ens-lyon.fr/pdf/ses/chatel.pdf

Chesnais (François) dir., La mondialisation financière, Paris, Syros, 1996.

Chevallard (Yves), Questions vives, savoirs moribonds : le problème curriculaire aujourd'hui, Communication au colloque Défendre et transformer l'école pour tous, Marseille, 2, 4 et 5 octobre 1997 http://www.eloge-des-ses.fr/documents/chevallard-questions-vives.pdf

Collectif, Sociologie de la mondialisation, Actes de la recherche en sciences sociales, $n^{\circ} 151-152$, mars 2004.

Cordellier (Serge) dir., La mondialisation au-delà des mythes, Paris, La Découverte, 1997.

Dagorn (René), Une brève histoire du mot « mondialisation », in GEMDEV, Mondialisation. Les mots et les choses, Paris, Karthala, 1999.

FMI (2000), La mondialisation : Faut-il s'en réjouir ou la redouter ?, http://www.imf.org/external/np/ exr/ib/2000/fra/041200f.htm\#II (consulté le 22/08/2013).

Fourçans (André), La mondialisation expliquée à ma fille, Paris, Seuil, 2001.

GEMDEV, Mondialisation. Les mots et les choses, Paris, Karthala, 1999.

Hymer (Stéphen), La grande « corporation » multinationale, Revue économique, Vol. 19, n 6, 1968. 
Le Cacheux (Jacques), Mondialisation économique et financière : de quelques poncifs, idées fausses et vérités, Revue de l'OFCE, Hors-série, mars 2002.

Lecler (Romain), Sociologie de la mondialisation, Paris, La Découverte, Coll. Repères, 2013.

Levitt (Theodor), The Globalization of markets, Harvard Business Review, Mai-juin 1983.

Martin (Denis) et alii, Les métamorphoses du monde. Sociologie de la mondialisation, Paris, Seuil, 2003.

Mattelart (Armand), La diversité culturelle : entre histoire et géopolitique, Communication au colloque Globalisme et pluralisme, Montréal, 24-27 avril 2002. http://www.er.uqam.ca/nobel/gricis/actes/ bogues/MattelarA.pdf (consulté le 25 août 2013).

Mattelart (Armand), Diversité culturelle et mondialisation, Paris, La Découverte, Coll. Repères, 2005.

Ohmae (Kenichi), Triade Power. The Coming Shape of Global Competition, The Free Press, 1985

(traduction française, La triade. Emergence d'une stratégie mondiale de la puissance, Paris, Flammarion, 1985).

Palloix (Christian), L'économie mondiale capitaliste, Paris, Maspéro, 1971.

Pisani-Ferry (Jean), Le réveil des démons, Paris, Fayard, 2011.

Reich (Robert), The Work of Nations, Vintage Press, 1991 (traduction française : L'économie mondialisée, Dunod, 1993).

Sassen (Saskia), La ville globale, Bruxelles, Desclée de Brouwer, 1996.

Sassen (Saskia), La globalisation, une sociologie, Paris, Gallimard, 2009.

Siroën (Jean-Marc), L'économie mondiale, Paris, Armand Colin, 1994.

Wagner (Anne-Catherine), Les classes sociales dans la mondialisation, Paris, La Découverte, Coll.

Repères, 2007.

\section{NOTES}

1. Cette discipline, créée en 1967, n'est enseignée que dans la filière B, puis ES de l'enseignement général. Elle a pour vocation de former les élèves a un certain nombre de savoirs issus de la science économique, de la sociologie et de la science politique.

2. Professeur de sciences économiques à la Sorbonne, M. Byé est l'auteur d'un manuel de référence dans le domaine de l'économie internationale. La publication de ce manuel sera poursuivie par G. Destanne de Bernis.

3. Il renvoie notamment en France aux publications d'Armand Mattelart qui seront largement utilisées dans les manuels pour traiter ce point du programme (Mattelart, 2002 et 2005).

4. Par exemple, lors de la mise en place des actuels programmes, les points les plus vivement contestés par une partie des enseignants correspondaient aux questions les plus nouvelles par rapport aux anciens programmes (sociologie des réseaux sociaux et économie du développement durable par exemple). Alors même que ces questions constituaient à la fois des avancées importantes dans les discipline de référence et des savoirs utiles pour comprendre un certain nombre de débats publics.

5. Rappelons que J. Le Cacheux était membre du groupe d'experts qui a rédigé les programmes du début des années 2000 et président du groupe d'experts qui a rédigé les programmes des années 2011-2012.

6. Sur les questions vives et les SES, je me permets de renvoyer à mon texte : http://www.elogedes-ses.fr/pages/textes-en-ligne/qsvconfusions-ab-2004.pdf, sur la mondialisation comme 
question vive, on consultera la contribution d'Alain Legardez : « La mondialisation, une question vive émergente dans l'enseignement et dans la formation » http://www.ices.fr/BU/documents/ koha_99956/pdf/a5_/legardez_alain_2.pdf

7. Le livre d'André Fourçans (2001) est un bon exemple de cette approche apologétique qui use de la référence à la science économique pour légitimer un discours économique caricaturalement libéral.

8. Il existe en effet un discours, porté notamment par l'association professionnelle, certains syndicats, diffusé dans des lieux de formation et assez généralement non discuté qui met l'accent sur le refus de la spécialisation disciplinaire, la pédagogie inductive, la critique du savoir économique, etc. Pour une présentation critique de cette doxa professionnelle voir Beitone (2013).

9. L'un des premier manuel de SES, dirigé par Jean Ibanes aux éditions Bordas, comportait d'ailleurs une série de volumes spécialisés : le changement social était traité par le sociologue Jean-Claude Chamboredon et la monnaie par l'économiste Huguette Durand.

10. Notamment en France. Par exemple le livre d'Appadurai parait aux Etats-Unis en 1990, il n'est traduit en français qu'en 2005.

11. Rappelons-le, la mondialisation est aussi un objet d'étude pour l'histoire et la géographie, disciplines scolaires qui sont enseignées parallèlement aux SES. Il ne s'agit donc pas, pour chaque discipline scolaire, d'appréhender l'objet dans sa totalité (ce qui est d'ailleurs impossible) mais de l'appréhender du point de vue spécifique de telle ou telle discipline. La mondialisation du géographe n'est pas la même que la mondialisation de l'économiste ou du sociologue, car chaque discipline construit ses objets d'études à partir de ses concepts et de ses traditions théoriques propres.

12. Le texte d'Yves Chevallard, Questions vives, savoirs moribonds : le problème curriculaire aujourd'hui (1997), reste une référence incontournable.

\section{RÉSUMÉS}

L'article présente tout d'abord une réflexion sur le rythme de l'introduction du concept de mondialisation dans les programmes de Sciences économiques et sociales de l'enseignement général en France. Il montre que, conformément à la théorie de la transposition didactique, il s'écoule un temps relativement long entre le développement d'un concept dans les savoirs savants et son introduction dans les savoirs scolaires. Dans un second temps, l'article examine à propos du cas de la mondialisation, certains débats qui traversent l'enseignement des sciences économiques et sociales: le débat sur le traitement des "questions vives " en SES, le sur le rapport entre les disciplines de référence de cette disciplines scolaire, enfin le débat sur le degré de complexité (ou d'ambition) des programmes.

The paper first presents a reflection about the pace of the introduction of the concept of globalization in the economic and social sciences programs in general education in France. It shows that, according to the theory of educational transposition, it takes a relatively long time between the development of a concept in the academic knowledge and its introduction into school knowledge. In a second step, the paper discusses about the case of globalization, some debates that the teaching of economics and social sciences: the debate on the treatment of 
"central issues" in SES, the relationship between the disciplines reference this school disciplines, and finally the debate on the degree of complexity (or ambition) programs.

INDEX

Mots-clés : mondialisation, programmes scolaires, sciences économiques et sociales

Keywords : economic and social sciences curricula, globalization

\section{AUTEUR}

\section{ALAIN BEITONE}

Professeur de sciences économiques et sociales au lycée Thiers (Marseille), Formateur à l'ESPE d'Aix-Marseille. Ex membre des deux groupes d'experts qui ont rédigé les dernières générations de programmes de SES 